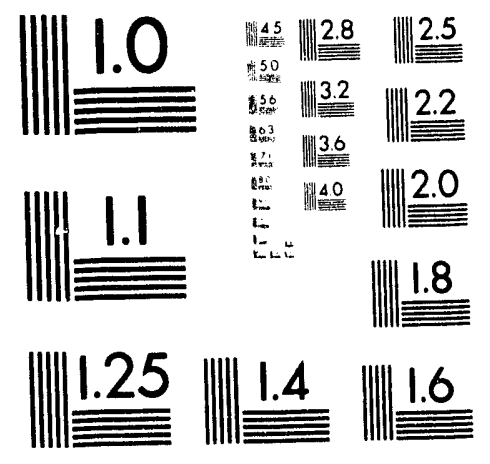



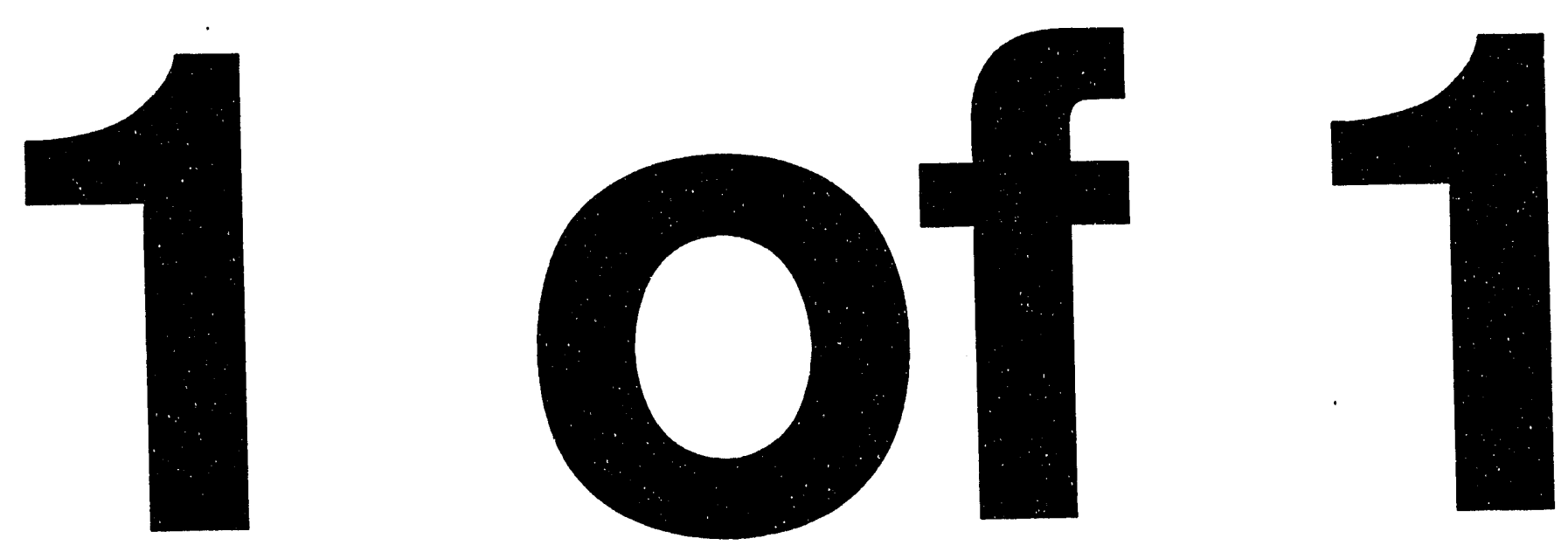


\title{
A Study of the Potential for Cooperation Between Models and Modelers
}

\author{
S. S. Stevens \\ B. E. Tonn \\ F. Southworth
}

Prepared by the

OAK RIDGE NATIONAL LABORATORY

Oak Ridge, Tennessee 37831-6207

managed by

MARTIN MARIETTA ENERGY SYSTEMS, INC.

for the

U.S. DEPARTMENT OF ENERGY

under contract DE-AC05-84OR21400

Presented At:

1993 Conference on Computing in the Social Sciences

National Center for Supercomputing Applications

University of Illinois

May 18-21, 1993

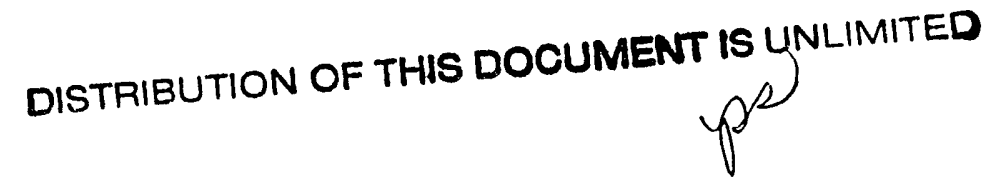

"The submitted manuscript has been

authored by a contractor of the U.S.

Government under contract No. DE-

ACO5-84OR2 1400. Accordingly, the U.S.

Government retains a nonexclusive,

rovalty-free license to publish or reproduce

the published form of this contribution, or

allow others to do so, for U.S. Government

purposes." 


\section{BACKGROUND}

Models describe aspects of nature or culture. They can be symbolic, analogue, visual and/or digital. Some models represent an accumulation of knowledge about nature. Other models are designed to simulate aspects of nature. Models play significant roles in many scientific endeavors and form the heart of most sophisticated analyses of government economic, health and other social welfare programs.

The use of computer programs to imitate nature is a popular technique at Oak Ridge National Laboratory (ORNL) for seeking answers to complex questions. Modeling is the tool employed when the underlying phenomena are believed to be well represented by a known set of equations, but the interesting large-scale problem is too complex to seek a closed, symbolic solution, and a physical experiment is either impossible or infeasible. In such cases, the governing equations are coded, initial conditions are specified, and an iterative numerical solution is computed. A useful extension of the preceding scenario is to specify rules for creating initial conditions, and let the program generate starting conditions. In any case, computer simulation is substituted for actual experimentation. Programming and generation or retrieval of the starting conditions take the role of experiment design, and the program's outputs are the experimental results.

The current explosion in the availability of desk top computing power probably is a major contributor to the pervasiveness of modeling. Certainly at ORNL many models spanning the range of desk top computing power are now in use. Most of the dozen or so modelers interviewed for this study prefer to work on their desk top machines, although some larger models are designed to run on supercomputers under some circumstances. (An interesting note from the interviews -- the size of the output from models discussed with us covers 15 orders of magnitude, from two numbers per run to terabytes of output data -- but the modeler expects his model to run in no rore than "minutes." Perhaps as much as any other characteristic, this establishes modeling at ORNL as a research tool.)

Many of the models now in use at ORNL are run in isolation by a small group of researchers to solve a particular problem. For models run in isolation, input and output formats can take any form that seems appropriate and convenient for the modeler and his audience.

At a higher level of complexity, researchers in some groups at ORNL use sets of models. Each model within the set attacks a sub-problem, and results from one model 
become starting conditions for the next. As long as the programmers who write the models can communicate with each other, it is feasible to shape outputs from one model into inputs suitable for the next.

As affordable desk top computing power becomes sufficient for models in many fields, one might project that the next requirement be to pass data among models not written by closely cooperating programmers. A researcher in one field might wish to draw his data from a model in a different field, just because both fields consider aspects of the same real-world entities. That step is not yet reality at ORNL, but it is a gleam in the eyes of a few researchers, here and elsewhere. This study will consider the state of the art of modeling at ORNL, and look at the prospects for co-operation among models from disparate fields of investigation.

This is an important topic for study not only from the viewpoint of the physical sciences but from the viewpoint of making ORNL science relevant for policy analysis exercises. For example, researchers from ORNL's Environmental Sciences and Energy Divisions have met on several occasions to discuss how ESD's scientific work might be able to complement the Energy Division's policy research related to global climate change. One conclusion of the participants is that ESD's models need to fit seamlessly with policy models (Rayner et al. 1992). If that fit is missing, in many cases good and potentially influential science will not make it into the policy process. Since the vast majority of models are computerized, an important step in ensuring that models communicate with each other is developing the computer science infrastructure to allow the models to communicate.

\section{SCOPE OF THIS STUDY}

In this study, we limited our questions to ORNL, discussing their models with almost a dozen staff members from four divisions. We collected some low-level data about the models, and also tried to gain a sense of the philosophy of the modeler, and how each model fit into the larger perspective of ORNL's and the scientific community's efforts. Time and budget prevented us from conducting any larger study, but we have no reason to suppose that conclusions about ORNL's models and modelers could not be extended to the larger scientific community. 


\section{CHARACTERISTICS OF MODELS AT ORNL}

Models, like other artifacts, are strongly influenced by the tools used to build them. At the most fundamental level, models are built with a programming language. The modelers we questioned were divided evenly between FORTRAN and C, with one researcher using compiled BASIC. The user-interface components of the mixed-language models were somewhat more likely to be written in $\mathrm{C}$, with the core computations in FORTRAN. Models now in active development are benefitting from the rich tool sets available to programmers; one model directly uses a commercial spreadsheet as its interface, and others use geographic information systems (GIS) for storage, preparation of input, or portrayal of output.

All the models discussed with us are designed to be able to run on desk top computers; the larger models -- written in C -- run primarily on UNIX workstations, and the rest run primarily on PC-compatible personal computers. Some models of global scope are of the sort that can expand to fill the available computing power; these models are being targeted at parallel processors here at ORNL and elsewhere. Some models in the robotics program here may have real-time applications, and can run -- very quickly -on application-specific integrated circuits. A couple of the modeling groups are designing their products to run on many different computers. Nonetheless, in spite of the potential variety of platforms, we get a strong sense that most of the research with ORNL models is carried out on desk top machines.

Only a couple of the models are designed to allow users to interact with the program as it runs. The rest are essentially batch operations, depending on the quick runtime turnaround for interactıvity.

Most of the models have explicit ASCII files as the "input" and "output," and the older models have limited tools to aid in either the construction of inputs or the interpretation of outputs. However, the model written in BASIC uses a coupled spreadsheet for data entry and display of results, taking advantage of the popular and intuitive user interface of the commercial product.

Several models now being developed utilize GIS for input/output, taking advantage of the representational capabilities of these special purpose data managers. Staff within the Energy Division's Center for Transportation Analysis are playing a leading role in the development of geographic data standards associated with the creation 
and dissemination of ground transportation network databases. Their work has revealed real stresses within the data-using community, at ORNL and elsewhere, in two important areas: (1) data accuracy, and (2) dynamic database management. Both areas will require work if we are to pass data with regularity from one modeling (sub)system to another. Data accuracy in a GIS context usually means putting a point or line on the right part of a map or computer screen. Few errors are so readily apparent, and so damaging to a (possibly otherwise superb) analysis than a map with obvious locational errors. Likewise, dynamic database management (data updating according to set protocols, with potential damage controls) will become more important as we move steadily into the era of near real time data collection and exchange.

In general, the models in use at ORNL either are isolated -- do not interact at all with other models -- or are run as part of a closely coupled set. However, there are at least two instances where modelers are actively seeking to link previously unrelated models. At the large-model end of the spectrum of activity, global change modelers in Environmental Sciences Division have received significant funding to assist Lawrence Livermore Laboratory in the linking of a number of previousiy independent global models. The effort is expected to result in model-specific code that alters input and output file specifications. Locally, modelers in Energy Division are attempting to link a set of models that consider the results of toxic chemical release and steps that can be taken by public officials to mitigate undesirable effects. In the Energy Division model linking work, the modelers hope to use off-the-shelf software to create a more general coupling process.

\section{STRATEGY FOR LINKING MODELS -- WHY, WHEN, HOW?}

It is reasonable to ask why models should be linked. The easy answer is "to solve more complex problems." Perhaps a better answer can be phrased as another question: "Can we decompose complex problems into sub-problems and thereby make them easier to understand and solve?" The answer to the second question is almost certainly "yes," especially when the problems we wish to consider have national or global scope, and contain aspects that should be addressed by various disciplines, including both physical and social scientists. Models should be coupled, then, if their cooperation results in better science, engineering or policy-making. 
If we can agree that models should sometimes be linked, it is also reasonable to ask when -- under what circumstances or conditions -- they should be linked. Before we can answer the imperative, we must establish the possible. It is possible to link two models when they both consider one or more of the same aspects of an entity -- when they're imitating overlapping parts of the world. An example is illustrative: the program that models traffic flow during evacuation of an area that is threatened by a toxic chemical release considers the same geographical area that is covered by a plume dispersion model. Better traffic engineering and public warning policy can result if the two models can easily be linked. One would like to adjust traffic signals to route the public away from parts of the area that are about to be covered by the toxic release. Models should be coupled, then, when they address common aspects of the modeled world, and their cooperation results in better science, engineering or policy-making.

"Why?" and "When?" are contemplative questions. "How?" is action oriented. In the remainder of this report, we will consider in some detail how models can be led to cooperate. To the question "How can models cooperate?", we propose the short answer "Use a common representation of common data." That answer will lead us into more-orless detailed discussions of data representation and the state of computing technology and art.

\section{SOFTWARE TOOLS AND TECHNIQUES}

The American Standard Code for Information Interchange -- ASCII -- is perhaps the most elementary level at which computer users attempt to understand the machine representations of their data. Every modeler we interviewed said that "ASCII files" were the basic input/output units for his model. That implies that the models all use positional representation for their data; that is, the programs expect to find their input in specific locations within sets of stored characters (ASCII files). The accessing functions are like FORTRAN's COMMON capability, with its advantages and its pitfalls. The burden of verification of data passed to a function or input into a model remains with the programmer, and close collaboration is necessary for efficient information passing between programs. 


\section{Separating Models from Data}

Experience with writing a number of complex, multi-component software systems suggests that an important design consideration for the development, portability and expandability of software is that of "data encapsulation". Energy Division staff and coworkers at the University of Tennessee have recently addressed this problem by developing systems which separate the data from the mathematical models in such a way that the data can be updated and the models revised independently of one another.

This approach to system design means that the mathematical routines are encapsulated and are separated from the data being used. Similarly, each element of the database, or data library, and of the user interface are also encapsulated and "hidden" from the mathematical algorithms and from each other. Figure 1 illustrates this idea of data encapsulation. In essence, a series of layers of functions are created in the program code. At the highest level are the mathematical algorithms which address the scientific problem. These routines obtain data via a set of Data Interfacing Functions (DIFs) which hide the location and format of the data from the algorithms. These DIFs access internal data structures directly but do not deal with input and output at the disk level. All disk Input/Output is achieved by the Data Manager, routines which access disk files and provide memory management functions. Program control is achieved through the use of a run (experiment)-specific file (termed a Scenario Configuration File in Figure 1) which, in conjunction witil the data, provides the parameters necessary to control and direct the individual mathematical algorithms. The advantages of such a design are as follows (Ray, 1992):

- during a port to other computer platforms only the routines that provide input and output functions need to be modified to accommodate differences in file naming conventions and directory hierarchies.

- alternative databases and user interfaces can be readily installed by a programmer who does not necessarily need to be familiar with the mathematical models.

- data interfacing, algorithms, and code structure, can be standardized to achieve a highly maintainable and portable system. 
- the mathematical algorithms can be reduced to a fundamental level, which further facilitates maintenance and extension.

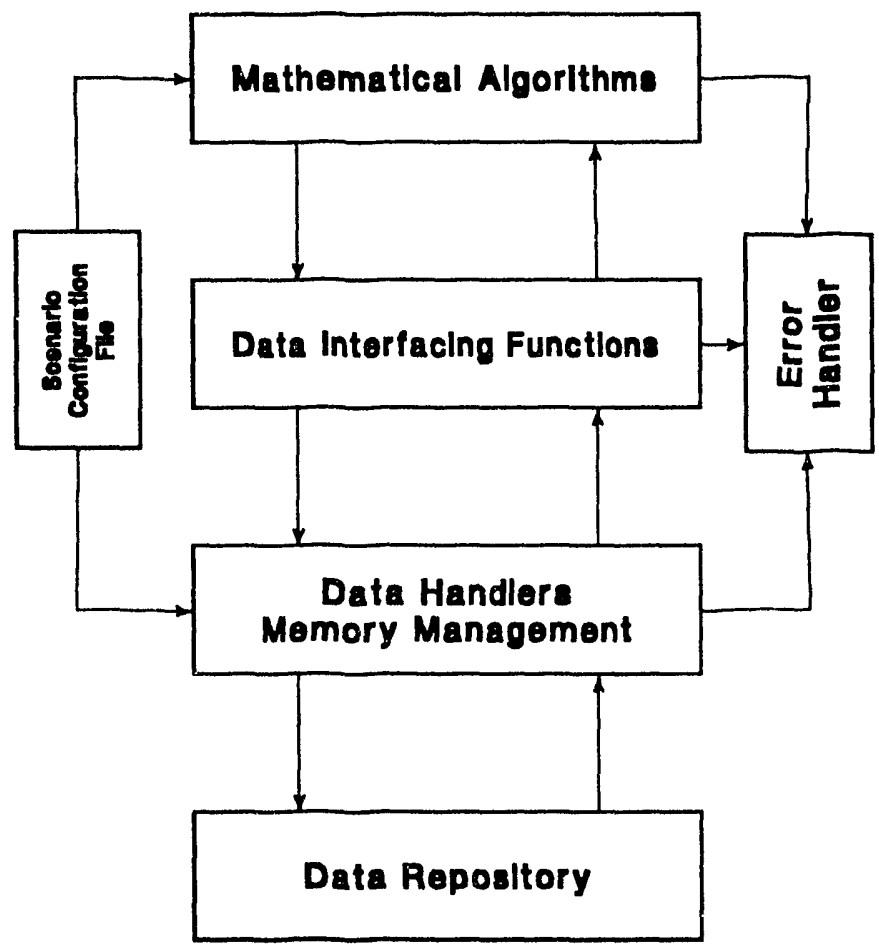

Figure 1 Data encapsulation

Taken together these traits render modelers more capable of meshing their code with other systems, and on such systems' hardware platforms. Divorcing the models from the data in this manner is also a positive step towards linking models from different systems within a single data model. That is, either a single data model can be promulgated from the start as a basis for subsequent systems integration; or the data from one system can be re-modelled to fit the second system, without having to also redesign the algorithms. 


\section{Non-Procedural Languages}

As we have mentioned, the most fundamental tool in common use by programmers is the language in which they write (FORTRAN, C or BASIC for the models discussed with us). For the past two decades, such "procedural" languages, where the programmer must explicitly describe detailed processes, have been dominant. As the power of available computers increases, much of the low level bookkeeping of writing computer programs is now shifting to the machines and language compilers. In many problem domains, there has been a significant shift to non-procedural languages, where the programmer describes what to do, as distinguished from how to do it. Structured Query Language (SQL) is perhaps the most widely used non-procedural language today, but programming language development is a dynamic field.

Data-centered application programming -- most busincss programming, for example -- emphasizes the demonstrable correctness of the data. To that end, an established, mature set of software tools, relational data base management systems (DBMS), is available to and used by business programmers. An essential feature of the latest generation of commercial DBMS -- relational DBMS -- is that data about entities are stored and can be retrieved by their attributes, without regard for their physical location. The programming language SQL can be used to store and retrieve data in such a DBMS without the need to know the internal structure of the data files. Furthermore, verification of data against predetermined correctness criteria can be performed automatically by the software tools.

\section{Object Orientation}

The most recent popular development in computer languages is object oriented programming. Objects are data entities that have the following characteristics: a class, attributes, inherited attributes, and instances. For example, a class could be employees. Attributes of employees might include age, education, sex, salary, and years of service. A second class could be scientific staff. This class is a subclass of employees. Therefore the class scientific staff inherits all of the attributes of the class employees and has additional attributes, such as discipline, patents awarded, and articles published in refereed journals.

A well designed object oriented database describes reality through its definitions of classes and subclasses. This description of reality should be holistic in the sense that 
modelers should be able to agree on how to represent reality in such a way as to define mutually acceptable objects. Once a mutually agreeable definition of objects is accomplished, then modelers would pick and choose objects from the collection for use in their models.

A shared object oriented database could contain instantiations of objects, either created through statistical analysis of primary data or output from models. For example, a plume model could update an instance of a class of objects known as atmospheric cubes. Atmospheric cubes could have attributes that define their precise location in the atmosphere and concentrations of various pollutants and other chemicals. A plume model could update a subset of attributes related to concentrations of toxics released from a toxic spill. An evaluation model could then tap the database to retrieve the updated atmospheric cubes to provide information abcut where toxics are likely to be.

The advantage of using objects is that they are more or less free standing entities. One can change an attribute of an object without worrying about tracking down other changes in the database. Object oriented systems will automatically change inheritance of attributes when attributes are added or deleted. Also, once defined, it is not expected that the object class definitions will change much if at all. These characteristics make administering object oriented databases relatively easy.

Another advantage is the ability to make objects dynamic rather than just static data entities. Specifically, attributes can be designed to initiate actions that effect other objects. For example, in battle simulation, a warplane object can receive messages from and generate messages to other warplanes. The warplane objects also have rules in their attributes which guide their behaviors -- movements, strategy, and message generation. Thus, the exciting possibility is for object oriented models to exercise dynamic objects. The models could output changes to the object oriented database (i.e., to instances of objects) as the simulation progresses or at the conclusion of the simulation. Additional models could use these outputs and/or in a choreographed manner, also update the object descriptions as they are exercised.

\section{Multimedia}

Another promising software technology that is happening now is the emergence of multi-media presentations. Narrowly construed, "multimedia" is sound and animation invoked in a computer presentation, but the tools needed to store, process, and 
synchronize audio and video are just the sort of programmers' aids that could be used to select and present the aspects of real-world representations created and stored by one model, and used as input by another. In particular, multimedia tools emphasize data compression and expansion, and time registration of various pieces of data, both of which certainly are relevant techniques for modelers that consider large-scale or global phenomena. [We would like to acknowledge the contributions of Wolfgang Naegli in pointing out the relevance of Apple Computer's Qu:ckTime multimedia tool set.]

\section{Using the Tools for Cooperative Modeling}

Clearly, good programmers can take the available tools and create data representations that serve models at every state of the art. ORNL has a premier example of ASCII file representation, in the activities of the Carbon Dioxide Information Analysis Center. The Center staff are strongly proactive in the validation, maintenance and distribution of worldwide data about $\mathrm{CO}_{2}$ and related climatological data. CDIAC not only archives the data, but assesses (and often improves!) their quality, and furnishes them to interested researchers along with instructions and sample FORTRAN programs for their use.

Although we did not discover any working examples of the use of relational DBMS technology by modelers at ORNL, that technology is sufficiently mature to be used effectively by loosely coupled models. It should be straightforward to represent modeled aspects of the world in the Entity-Attribute paradigm, facilitating their storage and access with non-procedural software tools. The required software skills are available at ORNL, and Energy Division modelers are investigating such tools for use with their chemical release mitigation models. The common perception that relational data base technology is "slow" has been overtaken by advances in kernel DBMS capability and hardware speed. The desirable features of data representation in the relational data model can considerably enhance the accessibility and transportability of common data to be used by more than one model.

Object oriented programming and multimedia-like treatment of data are exciting possibilities for future work, but neither technology is yet well-enough defined to allow its use by cooperating modelers without explicit declaration of conventions. Nonetheless, both techniques have the potential to make major impacts on the modeling process. 


\section{MORE THAN JUST TECHNOLOGY}

Technology will greatly facilitate the cooperation of models but technology by itself will not ensure the most effective cooperation of models. There is another issue that must be addressed and that is data quality. Models that are sharing data, passing data to each oth cr, creating data to be used in other models, need to consider data quality issues. The problem is that cooperating models may be sharing, generating and passing data of dubious quality. If this is the case, the models should take this into account.

The conceptual problem is how to express data quality in a form that is generic to all data items and scientific estimates. The key to solving this problem is to develop a pedigree that can be attached to a piece of data that indicates its quality. The Energy Division has been working on such a pedigree, known as NUSAP (Schaffhauser, Tonn, and Cantor 199\%). NUSAP stands for Numeral, Unit, Spread, Assessment, and Pedigree. The numeral is the actual data point, estimate, etc. The unit is selfexplanatory (e.g., pounds, feet). Spread refers to lower and upper bounds around the numeral, indicating that the true numeral is in the range with a certain level of confidence. Assessment refers to how useful the numeral and spread are: Does the spread effectively narrow the range of possibly true values? Will the value of the numeral decline over time? Can the value of the numeral be generalized to other cases? The pedigree contains descriptions of how rigorous the theory, data, and estimation methods were that created the datum. These aspects of the NUSAP approach allow uncertainty to be explicitly considered. NUSAP will be applied in a current ORNL U.S. Environmental Protection Agency project to estimate hazardous wastes generated for interstate commerce.

\section{POTENTIAL PROBLEMS AND OPPORTUNITIES}

Perhaps the most obvious threshold to be reached before there is widespread data sharing among models is fluency with the right software tools. For relational DBMS and Entity-Attribute representation, ORNL already has a body of talent and skill that can be tapped. For newer software techniques, either modelers must understand how to use the tools, or tool users must become part of the modeling team. 
It is possible that use of new software tools will impact the runtime of models and create reluctance to make more general representations of the modeled data. However, we believe that is more a problem of perception than of actuality, since available computer power continues to increase by almost a factor of two per year, and all but the largest models can be made to go faster just by paying a bit more for the hardware. In general, we believe that enhanced data accessibility and the serendipitous discoveries that may result from model cooperation will more than offset any degradation in runtime. Indeed, the time advantages to be realized in preparation of input and depiction of output from the use of general data manipulation software tools may well result in reductions in real turnaround time.

One of the traditional problems of automated data processing -- "garbage in, garbage out" or GIGO -- will remain as a problem, but we prefer to consider it an opportunity. The use of standard techniques for data representation will make it easier to address the concept of data quality. It will be a significant step forward when a stored data set carries with it an explicit representation of the quality of the data.

\section{PROGNOSIS}

Even this limited study of the prospects for cooperative modeling leads us to be quite optimistic about both the state of the art and the future. Certainly there are potential problems to be solved, both by individual modelers, and by the software community in general, but even existing tools are adequate to realize many advantages for modelers and their customers.

In the final analysis, cooperation among modelers is also a sociological problem. Modelers from different fields will have to talk to each other. They will have to find common jargon and negotiate the boundaries of each other's models. Technical tools are available to help in the realization of common concepts, but interpersonal communication may prove to be the most difficult challenge. We believe the benefits of increased communication across disciplinary lines will be worth the effort. 


\section{References}

Ray. J.J. and Southworth, F. (1992) Freight Network Policy Model. Highway Traffic Forecasting System Version 2.0B. User's Manual. Center for Transportation Analysis, ORNL/Transportation Center, University of Tennessee. February, 1992.

Ray J.J. (1992) Freight Network Policy Model. Technical Description. (DRAFT).

Transportation Center, University of Tennessee. November, 1992.

Rayner, S., Hillsman, E., Cushman, R., Farrell, M., Tonn, B., and Naegeli, W. 1992.

"Analyzing Sensitivity of Decisions to Global Climatic Change" Oak Ridge National Laboratory (draft)

Schaffhauser, A., Tonn, B., and Cantor, R. 1992. "Signaling the Uncertainty, Informative Value, and Credibility of Scientific Estimates for Policy" (submitted for publication) 

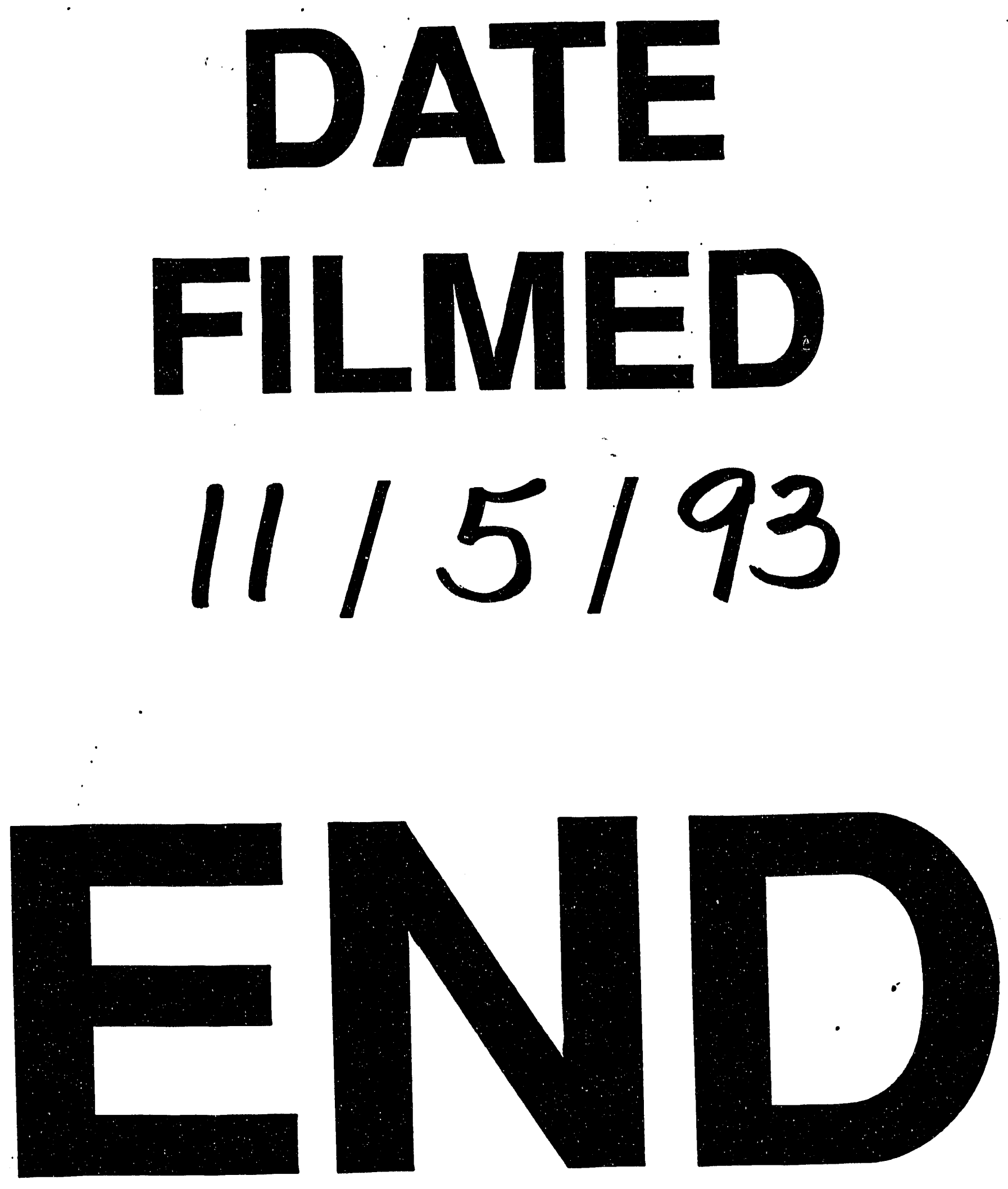
